\title{
Wie nachhaltig sind die gesetzliche Kranken- und Pflegeversicherung finanziert?
}

\author{
Sollten die Ausgaben der gesetzlichen Kranken- und der sozialen Pflegeversicherung \\ langfristig stark steigen, werden sowohl die jüngere Generation durch höhere Beiträge als \\ auch die Älteren durch mögliche Leistungseinschränkungen belastet. Auf Grundlage einer \\ neueren nichtparametrischen Schätzung wird eine Simulation der zukünftigen Entwicklung der \\ Beitragssätze in den beiden Zweigen der deutschen Sozialversicherung vorgestellt. Abhängig \\ von verschiedenen Annahmen über das künftige Wachstum des BIP pro Arbeitnehmer ergibt \\ sich dabei ein Gesamtsozialversicherungsbeitragssatz bis 2040 von nahe 50 \%. Damit ist die \\ Tragfähigkeit des deutschen Sozialversicherungssystems stark gefährdet.
}

Auf die Beitragszahler in der deutschen Sozialversicherung kommen durch den demografischen Wandel in den nächsten Jahrzehnten erhebliche Belastungen zu. Auch wenn die von der Bundesregierung eingesetzte Rentenkommission davor mehrheitlich die Augen verschlossen hat, steht ein dramatischer Anstieg des Beitragssatzes zur gesetzlichen Rentenversicherung bevor. Die in letzter Zeit regelmäßig aktualisierten Hochrechnungen von Martin Werding (Werding, 2018; Werding, 2019; Werding und Läpple, 2019) zeigen, dass bei Fortschreibung der „Haltelinie“ für das Rentenniveau bei $48 \%$ des Bruttolohns bereits 2040 dieser Beitragssatz die $25 \%$-Marke überschreiten würde, wenn die heute geltenden Regelungen bezüglich Regelaltersgrenze, Bundeszuschuss etc. ansonsten fortgeschrieben werden. Bezüglich der sonstigen Zweige der Sozialversicherung errechnet Werding in seinen Simulationen ebenfalls erhebliche Anstiege: So wird der Gesamtbeitragssatz zu den vier Sozialversicherungen gesetzliche Rentenversicherung (GRV), gesetzliche Krankenversicherung (GKV), soziale Pflegeversicherung (SPV) und Arbeitslosenversicherung (ALV) gemäß der Referenzvariante 2040 schon 48,8\% betragen, also knapp 9 Prozentpunkte mehr als heute.

In der öffentlichen Diskussion spielen die Beitragssätze zur Kranken- und Pflegeversicherung allerdings kaum eine Rolle, obwohl auch in diesen ein erhebliches Steigerungspotenzial schlummert. Bei der Krankenversicherung wurden vor allem zwei Faktoren identifiziert, die zu einem Anstieg der Pro-Kopf-Ausgaben beitragen können: zum

(c) Der/die Autor(en) 2020. Open Access: Dieser Artikel wird unter der Creative Commons Namensnennung 4.0 International Lizenz (https:// creativecommons.org/licenses/by/4.0/deed.de) veröffentlicht.

Open Access wird durch die ZBW - Leibniz-Informationszentrum Wirtschaft gefördert. einen die Alterung der Bevölkerung infolge steigender Lebenserwartung und geringer Geburtenzahlen und zum anderen der medizinische Fortschritt, der sich vor allem durch die Entwicklung neuer, aber teurer Behandlungsmethoden auszeichnet. Ob der Anstieg der Lebenserwartung tatsächlich die Gesundheitsausgaben erhöht, ist in der Literatur umstritten. Ein Gegenargument, das als Red-Herring-Hypothese bekannt geworden ist und auf Fuchs (1984) und Zweifel et al. (1999) zurückgeht, lautet, dass die hohen Ausgaben älterer Versicherter nichts mit ihrem Lebensalter selbst zu tun haben, sondern mit ihrer Nähe zum Tod, da die letzten Lebensjahre sehr behandlungsintensiv und damit teuer sind (für einen umfassenden Literaturüberblick Breyer, 2015). Da jeder Mensch aber nur einmal stirbt, würde ein Anstieg der Lebenserwartung die Sterberaten verringern und damit die durchschnittlichen Pro-Kopf-Gesundheitsausgaben eher senken.

In der Pflegeversicherung machen sich neben der demografischen Alterung vor allem die in den letzten Jahren eingeführten Leistungsverbesserungen sowie der drohende

Prof. Dr. Friedrich Breyer lehrt Volkswirtschaftslehre an der Universität Konstanz. Er ist Mitglied im Wissenschaftlichen Beirat beim Bundesministerium für Wirtschaft und Energie.

Prof. Dr. Normann Lorenz lehrt Volkswirtschaftslehre, insbesondere Sozial- und Verteilungspolitik/Gesundheitsökonomik, an der Universität Trier. 
Mangel an Pflegekräften bemerkbar, wobei letzterer eine bessere Entlohnung und damit höhere Kosten der Pflege nach sich ziehen wird.

\section{Eine neue empirische Analyse}

Um eine empirisch fundierte Voraussage zu treffen, wie sich die Ausgaben in der gesetzlichen Kranken- und Pflegeversicherung entwickeln könnten, wenn die institutionellen Regelungen, bezüglich der Generosität der Systeme, auf dem heutigen Stand erhalten blieben, bietet es sich an, die Ausgaben der jüngeren Vergangenheit zu analysieren und dabei den Versuch zu unternehmen, den Effekt der Altersstruktur von dem reinen Zeittrend zu trennen. Eine solche empirische Analyse wurde kürzlich von Lorenz et al. (2020) vorgelegt. Grundlage sind Inanspruchnahme-Daten einer Zufallsstichprobe von Versicherten der AOK Hessen, die von der PMV-Forschungsgruppe Köln aufgebaut wurde. Diese umfasst die Jahre 2001 bis 2015, pro Jahr ca. 300.000 Personen, deren Ausgaben für medizinische und Pflegeleistungen taggenau erfasst wurden (Ihle et al., 2005). Eine einmal gezogene Person bleibt so lange in der Stichprobe, bis sie verstorben ist oder die Versicherung aus anderen Gründen verlassen hat; es handelt sich also um ein unbalanciertes Panel. Bei Personen, die in diesem Zeitraum verstorben sind, ist ferner das Sterbedatum bekannt, sodass der Effekt der "Nähe zum Tod“ anhand der Ausgaben in den letzten zwölf Monaten des Lebens sowie in den vorangegangenen Zwölf-Monats-Abschnitten ebenfalls taggenau gemessen werden kann.

In der ökonometrischen Schätzung wurde der Zusammenhang zwischen dem Lebensalter und der Höhe der jährlichen Ausgaben für Personen im letzten, vorletzten, drittletzten und viertletzten Lebensjahr (Gestorbene) und für alle anderen, die „Überlebende“ genannt werden, nicht-parametrisch, d.h. als sogenannte Ausgabenprofile bestimmt. Um sicher zu gehen, dass eine Person das jeweilige Kalenderjahr tatsächlich um mindestens vier Jahre überlebt hat, konnten für diese Gruppe die Daten für 2012 bis 2015 nicht verwendet werden (ebenso fürs viertletzte Lebensjahr die Daten für 2013 bis 2015 usw.). Um die Stichprobe für die Schätzung des Zusammenhangs zwischen dem Alter und den Gesundheitsausgaben möglichst zu vergrößern, wurden alle 15 Beobachtungsjahre berücksichtigt, wobei die Daten aus den früheren Jahren mit der durchschnittlichen alterungsbereinigten Wachstumsrate der Pro-Kopf-Ausgaben in der GKV insgesamt auf das Jahr 2015 ,hochinflationiert" wurden. 'Die daraus bestimmten Wachstumsraten

1 Die dazu verwendeten altersspezifischen Ausgaben der Jahre 2001 bis 2015 entstammen der Datengrundlage des Bundesversicherungsamtes zur Berechnung des Risikostrukturausgleichs. dienen in einem zweiten Schritt dazu, den „reinen“ Zeittrend der Ausgaben zu ermitteln, den die Autoren als Ausdruck des medizinischen Fortschritts interpretieren. ${ }^{2}$

In der nicht-parametrischen Schätzung zeigt sich, dass in der Gruppe der Überlebenden die jährlichen Ausgaben für medizinische Leistungen etwa vom 40 . bis zum 80 . Lebensjahr steil ansteigen und danach etwas absinken. Bezieht man die Ausgaben der Pflegeversicherung mit ein, so steigen die Ausgaben auch im hohen Alter steil an: Im 90. Lebensjahr sind die Pro-Kopf-Kosten für Pflege höher als die für medizinische Leistungen. Für Gestorbene sieht das Bild vollkommen anders aus: sehr hohe Ausgaben, wenn der Tod zwischen 40 und 70 Jahren eintritt, in höherem Alter ein steiler Abfall, so dass die Ausgaben im letzten Lebensjahr eines 90-jährigen Gestorbenen weniger als ein Drittel der Ausgaben eines 70-jährigen Gestorbenen ausmachen.

\section{Simulationsergebnisse der Ausgabenseite}

Die so ermittelten Ausgabenprofile für GKV- und SPVAusgaben, getrennt nach dem Überlebensstatus, wurden im nächsten Schritt als über die Zeit konstant angenommen und auf die Altersstruktur der Jahre 2020, 2030, 2040 und 2050 entsprechend der 14. Koordinierten Bevölkerungsvorausberechnung des Statistischen Bundesamts angewendet, um den rein demografischen Effekt auf die Gesundheits- und Pflegeausgaben zu ermitteln. Dazu wird die Variante 2 (G2, L2, W2) angewendet, also konstante Geburtenrate bei 1,55 Kindern je Frau, Lebenserwartung bis 2060 ansteigend auf 84,4 Jahre für Männer und 88,1 Jahre für Frauen und eine Nettozuwanderung von 221.000 Personen im Jahr. Im Ergebnis steigen die GKV-Ausgaben pro Kopf nur aus demografischen Gründen bis zum Jahr 2050 um 6,8\%, die SPV-Ausgaben pro Kopf jedoch um 54,3\% und die Summe beider Größen um 12,7\%, alles verglichen mit dem letzten Jahr der Schätzung, 2015. Man kann daraus schließen, dass die „Red-Herring-Hypothese“, nach der der demografische Wandel die Gesundheitsausgaben nicht steigern werde, in Bezug auf die GKV-Ausgaben annähernd, wenn auch nicht buchstäblich bestätigt, in Bezug auf Pflegeausgaben jedoch klar widerlegt wird: Eine Veranderthalbfachung in 35 Jahren ist ein nennenswerter Anstieg.

In diesen Zahlen ist der reine Zeittrend noch nicht enthalten, der sich für die beiden Teilsysteme wie folgt errechnen lässt: In den 14 Jahren von 2001 bis 2015 stiegen die altersbereinigten Pro-Kopf-Ausgaben in der GKV um 44,5\%, die

2 Bezüglich der Ausgaben für Pflege existieren keine entsprechenden Daten zu den altersspezifischen Ausgaben in jedem Jahr für die gesamte SPV. Daher wurden diese Werte aus der vorliegenden Stichprobe der AOK-Hessen-Versicherten bestimmt und für die Inflationierung der Pflegeausgaben für 2001 bis 2014 auf das Jahr 2015 verwendet. 
Tabelle 1

Steigerungsfaktoren der Gesundheits- und Pflegeausgaben, bezogen auf 2015

\begin{tabular}{cccc} 
Jahr & Gesundheitsausgaben & Pflegeausgaben & Gesamtausgaben \\
\hline 2020 & 1,104 & 1,187 & 1,113 \\
\hline 2030 & 1,334 & 1,639 & 1,368 \\
\hline 2040 & 1,625 & 2,265 & 1,696 \\
\hline 2050 & 1,945 & 3,303 & 2,095 \\
\hline
\end{tabular}

Quelle: Lorenz et al. (2020).

altersbereinigten Pro-Kopf-Ausgaben für Pflege im AOKDatensatz um 54,2\%, während der Verbraucherpreisindex (VPI) im selben Zeitraum um 13,7\% zunahm. Inflationsbereinigt stiegen also die GKV-Ausgaben pro Kopf in diesen 14 Jahren um 27,1\% $(1,445 / 1,137-1=0,271)$, was einer konstanten Jahresrate von $1,73 \%$ entspricht, die Pflegeausgaben pro Kopf hingegen um 35,6 \% (1,542/1,137 - 1 $=0,356)$ oder mit einer Jahresrate von 2,20\%. Wendet man diese jährlichen Steigerungsraten auf die zuvor genannten rein demografisch hochgerechneten Ausgabenanstiege bis zu den Jahren 2020 bis 2050 an, so erhält man die in Tabelle 1 aufgeführten Steigerungsfaktoren. Man erkennt, dass sich unter den getroffenen Annahmen die Ausgaben für medizinische Behandlung bis 2050 (im Vergleich zu 2015) real fast verdoppeln, die Pflegeausgaben sogar verdreifachen werden, sodass die Summe beider Ausgaben sich real etwas mehr als verdoppeln wird.
Als ersten Test für die Prognosekraft des hier vorgestellten Simulationsmodells sei die tatsächliche Entwicklung der GKV- und SPV-Ausgaben pro Kopf von 2016 bis 2019 betrachtet, die in Tabelle 2 dargestellt ist. Es zeigt sich, dass das Simulationsmodell die wirkliche Ausgabenentwicklung in der GKV leicht überschätzt. Die tatsächliche jährliche Wachstumsrate war mit 1,67\% etwas geringer als die vom Simulationsmodell geschätzte von $2,00 \%$. Dagegen war die tatsächliche Entwicklung der Pflegeausgaben mit einer durchschnittlichen Jahresrate von $10,37 \%$ erheblich stürmischer als die geschätzte von $3,47 \%$, was leicht zu erklären ist: Diskretionäre Anpassungen (in diesem Fall: Ausweitungen) im Leistungsrecht lassen sich mit einem kontinuierlichen Prognosemodell nicht vorhersagen. Die absoluten Zahlen zeigen jedoch, dass die tatsächliche Ausgabenentwicklung in der Summe zu vorsichtig geschätzt wurde: Im Zeitraum 2015 bis 2019 steht einer Überschätzung der jährlichen GKVAusgaben pro Kopf um 40 Euro eine Unterschätzung der SPV-Ausgaben pro Kopf um 105 Euro gegenüber, und die jährliche Wachstumsrate der Summe wird um fast einen halben Prozentpunkt unterschätzt.

\section{Entwicklung der Einnahmeseite}

Aus der Entwicklung der Ausgaben einer Sozialversicherung allein - auch wenn man sie perfekt voraussagen könnte - lässt sich noch kein Urteil über die Nachhaltigkeit ihrer Finanzierung ableiten. Dazu muss man zusätz-

Tabelle 2

Entwicklung der Ausgaben für die Gesetzliche Krankenversicherung (GKV) und die Soziale Pflegeversicherung (SPV) 2015 bis 2019

\begin{tabular}{|c|c|c|c|c|c|c|}
\hline \multirow[b]{2}{*}{ Jahr } & \multirow{2}{*}{$\begin{array}{c}\text { Versicherte } \\
\text { in Mio. }\end{array}$} & \multirow{2}{*}{$\begin{array}{l}\text { Leistungs- } \\
\text { ausgaben } \\
\text { in Mrd. Euro }\end{array}$} & \multirow{2}{*}{$\begin{array}{l}\text { Verbraucherpreis- } \\
\text { index }\end{array}$} & \multicolumn{3}{|c|}{ Ausgaben } \\
\hline & & & & real & pro Kopf & Simulation \\
\hline 2015 GKV & 70,74 & 202,05 & 100,0 & 202,05 & $2.856,23$ & $2.856,23$ \\
\hline 2016 & 71,60 & 210,36 & 100,5 & 209,31 & $2.923,37$ & $2.913,32$ \\
\hline 2017 & 72,26 & 217,83 & 102,0 & 213,56 & $2.955,42$ & $2.971,54$ \\
\hline 2018 & 72,78 & 226,22 & 103,8 & 217,94 & $2.994,48$ & $3.030,92$ \\
\hline \multirow[t]{2}{*}{2019} & 73,01 & 234,58 & 105,3 & 222,78 & $3.051,31$ & $3.091,50$ \\
\hline & & & & Jahresrate & $1,67 \%$ & $2,00 \%$ \\
\hline 2015 SPV & 70,74 & 26,64 & 100,0 & 26,64 & 376,59 & 376,59 \\
\hline 2016 & 71,60 & 28,29 & 100,5 & 28,15 & 393,15 & 389,66 \\
\hline 2017 & 72,26 & 35,53 & 102,0 & 34,83 & 482,06 & 403,19 \\
\hline 2018 & 72,78 & 38,25 & 103,8 & 36,85 & 506,32 & 417,18 \\
\hline \multirow[t]{2}{*}{2019} & 73,01 & 41,27 & 105,3 & 39,19 & 536,81 & 431,66 \\
\hline & & & & Jahresrate & $10,37 \%$ & $3,47 \%$ \\
\hline 2015 GKV + SPV & 70,74 & 228,69 & 100,0 & 228,69 & $3.232,82$ & $3.232,82$ \\
\hline 2016 & 71,60 & 238,65 & 100,5 & 237,46 & $3.316,52$ & $3.302,98$ \\
\hline 2017 & 72,26 & 253,36 & 102,0 & 248,39 & $3.437,48$ & $3.374,73$ \\
\hline 2018 & 72,78 & 264,47 & 103,8 & 254,79 & $3.500,80$ & $3.448,11$ \\
\hline 2019 & 73,01 & 275,85 & 105,3 & 261,97 & $3.588,12$ & $3.523,16$ \\
\hline
\end{tabular}

Quelle: Statistisches Bundesamt und eigene Berechnungen. 
Tabelle 3

Beitragssatz-Projektion auf der Basis der BIP-Projektion des ifo

\begin{tabular}{|c|c|c|c|c|c|c|c|c|c|c|}
\hline \multirow[b]{2}{*}{ Jahr } & \multirow{2}{*}{$\begin{array}{c}\text { Einwohner } \\
\text { in Mio. }\end{array}$} & \multirow{2}{*}{$\begin{array}{c}\text { Erwerbstätige } \\
\text { in Mio. }\end{array}$} & \multirow{2}{*}{$\begin{array}{l}\text { BIP-Potenzial } \\
\text { in Mrd. Euro }\end{array}$} & \multicolumn{2}{|c|}{ BIP/Einwohner } & \multicolumn{2}{|c|}{ Ausgaben } & \multicolumn{3}{|c|}{ Beitragssatz (in \%) } \\
\hline & & & & in Euro & Index 2015=1 & GKV-Index & SPV-Index & GKV & SPV & Summe \\
\hline 2015 & 82,176 & 42,993 & 2807,948 & 34.170 & 1,000 & 1,000 & 1,000 & 15,50 & 2,43 & 17,93 \\
\hline 2020 & 83,392 & 45,537 & 3051,916 & 36.597 & 1,071 & 1,104 & 1,187 & 15,98 & 2,69 & 18,67 \\
\hline 2030 & 83,866 & 43,652 & 3298,598 & 39.332 & 1,151 & 1,334 & 1,639 & 17,96 & 3,46 & 21,42 \\
\hline 2040 & 82,968 & 41,297 & 3528,555 & 42.529 & 1,245 & 1,625 & 2,265 & 20,24 & 4,42 & 24,66 \\
\hline 2050 & 81,522 & 40,239 & 3848,738 & 47.211 & 1,382 & 1,945 & 3,303 & 21,82 & 5,81 & 27,63 \\
\hline
\end{tabular}

Quellen: Werding et al. (2020) und eigene Berechnungen.

lich die Entwicklung des Bruttoinlandsprodukts (BIP) kennen, um daraus Aussagen über die damit einhergehende Entwicklung der Beitragssätze treffen zu können. ${ }^{3}$ Für diese Abschätzung verwenden wir die folgende Identität:

$$
B S=\frac{\text { Ausgaben }}{B G}=\frac{\text { Ausgaben } / \text { Kopf }}{\text { BIP/Kopf }} \cdot \frac{B I P}{B G},
$$

wobei $B S$ für den Beitragssatz einer Sozialversicherung und $B G$ für dessen Bemessungsgrundlage (Summe der beitragspflichtigen Einnahmen) steht. Unterstellt man eine konstante Relation dieser Größe zum BIP (letzter Term in (1)), so muss man die Entwicklung des BIP/Kopf schätzen, um die gestellte Frage beantworten zu können.

Dafür wählen wir zwei unterschiedliche Vorgehensweisen:

1. Wir übernehmen eine Projektion des ifo-Instituts für den Fünften Tragfähigkeitsbericht des Bundesfinanzministeriums, und zwar die „mittlere" Variante, die ein konstantes Wachstum des BIP je Erwerbstätigen mit 1,18\% pro Jahr unterstellt (Werding et al., 2020). Die Werte ab 2020 finden sich in Tabelle 3.

2. Wir erstellen eine eigene Projektion, die auf der folgenden Identität beruht:

$$
\frac{B I P}{N}=\frac{B I P}{L} \cdot \frac{L}{E P P} \cdot \frac{E P P}{N}
$$

Darin bezeichnet $N$ die Bevölkerungszahl, $L$ die Zahl der Erwerbstätigen, EPP das Erwerbspersonenpotenzial, definiert als die Zahl der Einwohner in den Altersgruppen zwischen 20 und der Regelaltersgrenze. Ferner treffen wir die folgenden Annahmen: Die jährliche Wachstumsrate des BIP je Erwerbstätigen bleibe auf dem Niveau der

3 Die Auswirkungen der Corona-Pandemie auf das BIP 2020 und in den Folgejahren sind noch nicht berücksichtigt. vergangenen beiden Jahrzehnte (2000 bis 2019) konstant, in denen sie bei 0,61\% lag (Statistisches Bundesamt, 2020), die Erwerbsquote (L/EPP) bleibe konstant, und das Erwerbspersonenpotenzial als Anteil der Bevölkerung entnehmen wir der Variante 2 (G2, L2, W2) der 14. Koordinierten Bevölkerungsvorausberechnung des Statistischen Bundesamts und lesen den Quotienten EPP/N für das Jahr 2015 aus der Tabelle für die Altersgrenze 65 ab, für die Jahre ab 2030 aus der Tabelle für die Altersgrenze 67 und für 2020 aus dem Durchschnitt der Werte aus beiden Tabellen.

Vergleicht man die Werte in den beiden Tabellen, so fallen bezüglich der zeitlichen Entwicklung vom Ausgangsjahr 2015 an folgende Unterschiede auf:

- Gemäß den ifo-Zahlen (vgl. Tabelle 3) sinkt die Zahl der Erwerbstätigen bis 2040 um 3,94\% und bis 2050 um 6,28\%, während die Zahlen des Statistischen Bundesamts (vgl. Tabelle 4) bereits bis 2040 einen Rückgang um $8,62 \%$ und bis 2050 um 10,24\% voraussagen.

- Hinzu kommen die unterschiedlichen Annahmen über die Wachstumsrate des BIP je Erwerbstätigen (1,18\% p. a. bei ifo versus $0,61 \%$ p. a. in unserer Projektion).

- Als Konsequenz ergeben sich drastische Differenzen beim Wachstum des Pro-Kopf-BIP, welches das ifo mit $34,5 \%$ bis 2040 und $38,2 \%$ bis 2050 errechnet, während unsere Berechnungen nur $8,6 \%$ bzw. $17 \%$ ergeben.

\section{Entwicklung der Beitragssätze zur Sozialversicherung}

Entsprechend unterschiedlich - je nach Entwicklung des Pro-Kopf-BIP - fallen auch die Beitragssatzprognosen aus. Im Folgenden wollen wir uns auf 2040 konzentrieren, weil der Prognosefehler bei den demografischen Daten über einen 20-Jahres-Zeitraum nicht allzu groß sein sollte. Immerhin sind alle Menschen, die zum inländischen Erwerbsper- 
Tabelle 4

Beitragssatz-Projektion auf Basis der Annahme konstanten Wachstums des BIP je Erwerbstätigen

\begin{tabular}{|c|c|c|c|c|c|c|c|c|c|c|c|c|}
\hline \multirow[b]{2}{*}{ Jahr } & \multirow{2}{*}{$\begin{array}{c}\text { Einwohner } \\
\text { in Mio. }\end{array}$} & \multicolumn{2}{|c|}{$\begin{array}{l}\text { Erwerbspersonen- } \\
\text { potenzial (EPP) }\end{array}$} & \multicolumn{2}{|l|}{ BIP } & \multicolumn{2}{|c|}{ BIP/Einwohner } & \multicolumn{2}{|c|}{ Ausgaben } & \multicolumn{3}{|c|}{ Beitragssatz (in \%) } \\
\hline & & in \% & in Mio. & in Mrd. Euro & BIP/EPP & in Euro & $\begin{array}{c}\text { Index } \\
2015=1\end{array}$ & GKV-Index & SPV-Index & GKV & SPV & Summe \\
\hline 2015 & 82,176 & 61,0 & 42,993 & 2807,948 & 65.312 & 34.170 & 1,000 & 1,000 & 1,000 & 15,50 & 2,43 & 17,93 \\
\hline 2020 & 83,365 & 61,0 & 43,615 & 2936,524 & 67.328 & 35.225 & 1,031 & 1,104 & 1,187 & 16,60 & 2,80 & 19,40 \\
\hline 2030 & 83,341 & 58,3 & 41,673 & 2981,664 & 71.550 & 35.777 & 1,047 & 1,334 & 1,639 & 19,75 & 3,80 & 23,55 \\
\hline 2040 & 82,091 & 55,8 & 39,287 & 2987,258 & 76.036 & 36.390 & 1,065 & 1,625 & 2,265 & 23,65 & 5,17 & 28,82 \\
\hline 2050 & 80,200 & 56,1 & 38,589 & 3118,112 & 80.804 & 38.879 & 1,138 & 1,945 & 3,303 & 26,50 & 7,05 & 33,55 \\
\hline
\end{tabular}

Quellen: Statistisches Bundesamt (2020) und eigene Berechnungen.

sonenpotenzial jenes Jahres zählen, heute schon geboren. Ferner übernehmen wir bezüglich des GRV-Beitrags das Ergebnis der Referenzvariante aus Werding $(2018,31)$, nämlich $22,5 \%,{ }^{4}$ und unterstellen einen gleichbleibenden Beitragssatz zur Arbeitslosenversicherung von 2,4\%. Tabelle 5 fasst die Ergebnisse zusammen.

Man erkennt, dass selbst unter den relativ optimistischen Annahmen des ifo-Instituts bezüglich der Entwicklung des Erwerbspersonenpotenzials und des BIP je Erwerbstätigen der Gesamtbeitragssatz zur Sozialversicherung bis zum Jahr 2040 nahe an die 50\%-Grenze stößt - und das trotz einer zusätzlichen Belastung der Steuerzahler durch den gewachsenen Bundeszuschuss zur GRV im Umfang von 58 Mrd. Euro oder fast $2 \%$ des BIP. Legt man dagegen unsere Annahmen bezüglich beider Größen zugrunde, so müsste der zur Finanzierung aller Ausgaben erforderliche Gesamtbeitrag schon 2040 bei 53,7\% liegen. In beiden Fällen werden durch die Projektionen zwei Fragen aufgeworfen, die wir abschließend kurz diskutieren werden:

- Gibt es Maßnahmen im Rahmen der gesetzlichen Kranken- und Pflegeversicherung, mit denen die Beitragssatzentwicklung so rechtzeitig abgebremst werden könnte, dass die in Tabelle 5 präsentierten Zahlen nicht Wirklichkeit werden?

- Falls diese Maßnahmen ausbleiben, welche Generation wird dann in 20 Jahren als Verlierer dastehen: die (junge) Generation der Beitragszahler, weil sie eine so viel schwerere Abgabenlast zu tragen hat, oder die (alte) Generation, die den Löwenanteil der Leistungen aus der Sozialversicherung zu beziehen hofft, deren Leistungsansprüche jedoch zum Zweck der Stabilisierung der Beitragssätze drastisch beschnitten werden?

4 Anzumerken ist, dass dieser Beitragssatz unter der Annahme ermittelt wurde, dass der Bundeszuschuss zur GRV von heute $92 \mathrm{Mrd}$. Euro auf 150 Mrd. Euro steigt.
Mögliche Maßnahmen zur Stärkung der Nachhaltigkeit

In der GKV stehen folgende Optionen zur Verfügung, um den Anstieg des Beitragssatzes zu dämpfen (Breyer, 2015):

- Übergang zu kassenspezifischen Kopfpauschalen, mit denen die Beitragsbasis erweitert würde, insbesondere bei den Rentnern, die weitere Einkommen oder Vermögen haben;

- mehr Effizienz durch Stärkung des Wettbewerbs zwischen Krankenkassen und Leistungserbringern;

- verstärkte explizite Rationierung durch Eingrenzung des Leistungskatalogs auf Leistungen, die evidenzbasiert sind und Mindestgrenzen der Kosteneffektivität einhalten.

In der sozialen Pflegeversicherung wäre an eine obligatorische kapitalgedeckte Zusatzversicherung zu denken, die allerdings weniger die Ausgaben der SPV als die Zusatzkosten der Sozialhilfeträger dämpfen würde (Breyer, 2016).

Tabelle 5

Beitragssätze zur Sozialversicherung 2040

\begin{tabular}{lccccc} 
& GRV & ALV & GKV & SPV & gesamt \\
\hline ifo & 22,5 & 2,4 & 20,2 & 4,4 & 49,5 \\
\hline Eigene Berechnungen & 22,5 & 2,4 & 23,6 & 5,2 & 53,7 \\
\hline
\end{tabular}

Anmerkungen: GRV = Gesetzliche Rentenversicherung, ALV = Arbeitslosenversicherung, GKV = Gesetzliche Krankenversicherung, SPV = Soziale Pflegeversicherung.

Quellen: Werding et al. (2018) und eigene Berechnungen. 
Wer profitiert von mehr Nachhaltigkeit in der Sozialversicherung?

Wem nützt Nachhaltigkeit der Finanzierung eigentlich? Anders gefragt: Wer sind die Leidtragenden, wenn es bei fehlender Nachhaltigkeit zu einem Auseinanderklaffen von Leistungsansprüchen und Finanzierungsmöglichkeiten kommt? Sind es die nachfolgenden Generationen von Beitrags- und Steuerzahlern, die vor einer Überforderung geschützt werden sollen - worauf der Begriff der "Generationengerechtigkeit“" hindeutet? Oder sind es eher die „Alten", deren gesetzliche Ansprüche auf eine auskömmliche Rente, auf medizinische Behandlung nach dem Stand des Wissens und auf notwendige Pflegeleistungen dann nicht oder nur partiell erfüllt werden? Die Antwort hängt davon $a b$, ob der angesprochene Anstieg der Beitragssätze sowie die entsprechenden steuerfinanzierten Sozialleistungen das Parlament passieren oder ob dies politisch verhindert wird - mit der Folge, dass Leistungsversprechen zurückgenommen werden müssen.

Sinn und Übelmesser (2002) warnen, dass Reformen zulasten der Rentnergeneration in einer Mehrheits-Demokratie nicht mehr möglich seien, sobald diese die Mehrheit der wahlberechtigten Bürger stelle. Die Kosten einer nicht-nachhaltigen Finanzierung müssten demnach die späteren Generationen tragen. Die Gegenthese vertreten Breyer und Stolte (2001) mit dem Argument, dass die mittlere Generation, die das BIP erwirtschaftet, noch weitere Mittel als das Stimmrecht an der Wahlurne besitzt, um inre Interessen durchzusetzen (Rückzug in die Selbstständigkeit, politisch motivierte Streiks, Auswanderung). Und wenn eine Rentnergeneration, die mit der gesamten politischen Macht ausgestattet ist, diese tatsächlich so einsetzen sollte, dass sie die maximalen Sozialleistungen für sich erzielt, wird sie jenen Beitragssatz wählen, der das Beitragsaufkommen unter Beachtung der Abgabenwiderstände maximiert.

Daher werden nicht nur die jungen, sondern auch die alten Generationen den Schaden haben, wenn die Finan- zierung des Sozialsystems nicht nachhaltig ist. Deren gesetzlich verankerte Leistungsansprüche müssten dann wegen mangelnder Finanzierbarkeit zurückgeschraubt werden, so wie bereits in den Rentenreformen zwischen 2001 und 2007 zuvor bestehende Leistungsversprechen zurückgenommen wurden. Folglich sind es gerade die heutigen Beitragszahler und morgigen Rentner, die am meisten von Reformen zur Stärkung der nachhaltigen Finanzierung profitieren.

\section{Literatur}

Breyer, F. (2015), Demographischer Wandel und Gesundheitsausgaben: Theorie, Empirie und Politikimplikationen, Perspektiven der Wirtschaftspolitik, 16, 215-230.

Breyer, F. (2016), Die Zukunft der Pflegeversicherung in Deutschland: Umlage und Kapitaldeckung, Zeitschrift für die gesamte Versicherungswissenschaft, 105, 445-461.

Breyer, F. und K. Stolte (2001), Demographic Change, Endogenous Labor Supply, and the Feasibility of Pension Reform, Journal of Population Economics, 14, 409-424.

Fuchs, V. R. (1984), Though much is taken: Reflections on aging, health and medical care, Milbank Memorial Fund Quarterly/Health and Society, 62, 143-166.

Ihle, P. et al. (2005), Versichertenstichprobe AOK Hessen/KV Hessen - Konzeption und Umsetzung einer personenbezogenen Datenbasis aus der Gesetzlichen Krankenversicherung, Gesundheitswesen, 67, 1-8.

Lorenz, N., P. Ihle und F. Breyer (2020), Aging and Health Care Expenditure: A non-parametric approach, CESifo Working Paper, Nr. 8216, April.

Sinn, H. W. und S. Übelmesser (2002), Pensions and the path to gerontocracy in Germany, European Journal of Political Economy, 19, 153-158.

Statistisches Bundesamt (2019), Bevölkerung Deutschlands bis 2060, 14. koordinierte Bevölkerungsvorausberechnung.

Statistisches Bundesamt (2020), Volkswirtschaftliche Gesamtrechnungen, Fachserie 18; Reihe 1.5, Inlandsproduktberechnung Lange Reihen ab 1970.

Werding, M. (2018), Demographischer Wandel, soziale Sicherung und öffentliche Finanzen: Langfristige Auswirkungen und aktuelle Herausforderungen, Bertelsmann Stiftung.

Werding, M. (2019), Wie haltbar sind die Haltelinien? Effekte der Rentenreform 2018, ifo Schnelldienst, 72(2), 21-25.

Werding, M. und B. Läpple (2019), Wie variabel ist der demografische Alterungsprozess? Effekte von Geburten und Zuwanderung - Folgen für die soziale Sicherung, Bertelsmann Stiftung.

Werding, M., K. Gründler, B. Läpple, R. Lehmann, M. Mosler und N. Potrafke (2020), Modellrechnungen für den Fünften Tragfähigkeitsbericht des BMF, Studie im Auftrag des Bundesministeriums der Finanzen, ifo Studie.

Zweifel, P., S. Felder und M. Meier (1999), Ageing of population and health care expenditure: A red herring?, Health Economics, 8, 485-496.

Title: Is the Financing of German Health and Long-Term Care Insurance Sustainable?

Abstract: Based on a recent non-parametric estimation of the relationship between age, lifespan and health and long-term care expenditures in German social health insurance, we present a simulation of the future development of contribution rates in the two branches of the German social security system. Depending on different assumptions of the future growth of GDP per worker, our estimates show that by 2040 the total social security contribution rate could reach between slightly below $50 \%$ and above $53 \%$ of gross wages. Thus the sustainability of the German social security system is severely jeopardised.

JEL Classification: H51, J11, I19. 